\title{
MAP2 Expression and Neuritic Outgrowth and Branching Are Coregulated Through Region-Specific Neuro-Astroglial Interactions
}

\author{
B. Chamak, A. Fellous, ${ }^{1}$ J. Glowinski, and A. Prochiantz \\ Chaire de Neuropharmacologie, INSERM U.114, Collège de France, 75231 Paris Cedex 5, France, and 'INSERM U.96, \\ Hôpital de Bicêtre, F-94275 Le Kremlin Bicêtre Cedex, France
}

Embryonic neurons from the rat striatum and mesencephalon were plated on mesencephalic or striatal astrocytes in 4 possible combinations. It was found that specific traits are expressed by the neurons when they are grown on homotopic astrocytes (neurons and astrocytes from the same region). These traits are the following:

1. The number of cells stained with an antibody raised against the microtubule-associated protein 2 (MAP2) is higher in homotopic than in heterotopic cocultures. This is true for both mesencephalic and striatal neurons.

2. In homotopic conditions, there is an increase in the number of cells having more primary neurites and branching points. This effect is observed for both neuronal populations but is more pronounced in mesencephalic neurons.

3. The intensity of MAP2 staining was correlated with the branching ability of the neurons. First, on comparing MAP2positive and MAP2-negative cells, it was found that, in any combination (homotopic and heterotopic cocultures), the number of primary neurites and branching points was much higher in MAP2-positive cells. In fact, almost no branching activity was found in MAP2-negative neurons. Second, within the MAP2-positive neuronal population, the higher number of branching points observed under homotopic neuro-astroglial conditions was mostly due to the neuritic compartment, which was strongly and homogeneously stained with the anti-MAP2 antibody.

These observations strongly suggest that the astrocytic environment regulates the synthesis and/or intracellular distribution of MAP2, as well as the morphology of the neurons, and that this regulation is region specific.

The outgrowth of neurites is regulated by several factors in vitro. Some soluble factors have been partially or completely purified (Levi-Montalcini, 1982; Guenter et al., 1985; Kligman and Marshak, 1985; Walicke et al., 1986). Substrate adhesion molecules, molecules associated with substrate adhesion molecules, and cell adhesion molecules are probably involved in neuritic outgrowth since they mediate neurite-neurite or neurite-astrocyte

\footnotetext{
Received Sept. 11, 1986; revised Mar. 12, 1987; accepted Apr. 10, 1987.

We thank Dr. F. Rosa for his help in preparation of antibodies and Drs. G. Barbin, D. Katz, M. Mallat, and M. Murray for their critical and helpful suggestions.

This study was supported by grants from INSERM, Rhône-Poulenc Santé, and MRT (No. 84C 1315)

Correspondence should be addressed to A. Prochiantz, Chaire de Neuropharmacologie, INSERM U.114, Collège de France, 11 Place Marcelin Berthelot, 75231 Paris Cedex 5, France.

Copyright (C) 1987 Society for Neuroscience $0270-6474 / 87 / 103163-08 \$ 02.00 / 0$
}

interactions (Grumet et al., 1983, 1985; Rutishauser, 1983; Edgar et al., 1984; Smalheiser et al., 1984; Kruse et al., 1985; Lander et al., 1985; Stallcup and Beasley, 1985).

Recently, some attention has also been focused on signals that regulate the extent and branching of the neuritic arborization and its dendritic or axonal nature. Specific lectins can promote the outgrowth of branched or unbranched neurites (Chiquet and Acklin, 1986; De George and Carbonetto, 1986). Retinoic acid and dibutyril-cAMP induce the extension of branched and unbranched neurites, respectively, in a neuroblastoma cell line (Fischer et al., 1986). Satellite Schwann cells modify the neuritic morphology of dorsal root ganglia sensory neurons (Mudge, 1984). Finally, sympathetic neurons grown in vitro in the absence of satellite Schwann cells develop an axonlike unbranched neurite, while in the presence of Schwann cells a highly branched, dendrite-like microtubule-associated protein 2 (MAP2)-positive neuritic compartment can be observed (Allen and Higgins, 1986; Bruckenstein and Higgins, 1986).

These observations suggest that axonal and dendritic outgrowth might be differentially regulated. They also suggest that in some cases, as already proposed by Denis-Donini et al. (1984), specific neuro-astroglial interactions are involved in this regulatory process. Indeed, in a previous report we have shown that the morphological traits expressed by mesencephalic dopaminergic (DA) neurons differ depending on whether these cells are grown on their own local (homotopic) mesencephalic astrocytes or on striatal (heterotopic) ones. On heterotopic astrocytes most DA neurons display long, thin, and unbranched neurites, while on homotopic astrocytes several highly branched neurites develop. Preliminary ultrastructural studies have indicated that these 2 types of neurites may correspond to the dendrite-like and axon-like compartments characterized by the presence or absence of ribosomes, tapering constant-caliber neurites, and the shape of the branching points (Chamak et al., 1987).

In order to examine further the possibility that it is the interactions between neurons and homotopic astrocytes that specifically favor the outgrowth of a highly branched, dendrite-like compartment, we have analyzed the morphological traits of short-term cultures of mesencephalic and striatal neurons grown on mesencephalic or striatal astrocytic monolayers in the 4 possible combinations. In addition, we have studied the expression and distribution of MAP2 in these diverse neuro-astroglial cocultures. The choice of MAP2 was based on the fact that this protein, primarily associated with neurons, is highly enriched in the dendritic compartment in vivo and in vitro (Matus et al., 1981; Caceres et al., 1984, 1986; De Camilli et al., 1984; Peng et al., 1986). 
We demonstrate that neurons grown in homotopic neuroastroglial coculture are more complex (e.g., have more primary neurites and branching points) than cells grown in heterotopic conditions. In addition, it appears that neuronal expression of MAP2 is enhanced on homotopic astrocytes and is mainly associated with the highly branched neuritic compartment. We therefore propose that homotopic neuro-astroglial cocultures accelerate the outgrowth of a highly branched, MAP2-positive dendrite-like compartment through a specific but yet unknown mechanism.

\section{Materials and Methods}

Cell cultures. Astrocytes werc prepared as previously described (DenisDonini et al., 1984). Briefly, 15-d-old embryonic or postnatal day 4 rat mesencephalons and striata were dissociated in phosphate buffer, $\mathrm{pH}$ 7, complemented with D-glucose, $33 \mathrm{~mm}$ (PBS). Before dissociation in the presence of DNAse, $1 \mathrm{mg} / \mathrm{ml}$ (Sigma), postnatal tissues were incubated with $0.1 \%$ trypsin (Gibco). Dissociated cells were plated at the density of $5 \times 10^{4} / \mathrm{cm}^{2}$ on glass coverslips previously coated with 1.5 $\mu \mathrm{g} / \mathrm{ml} \mathrm{D,L-polyornithine} \mathrm{(Sigma).} \mathrm{Cells} \mathrm{were} \mathrm{cultured} \mathrm{for} 3$ weeks in an equal mixture of minimum essential medium and nutrient F1 2 (Gibco) supplemented with the following: glutamine, $2 \mathrm{~mm}$; glucose, $33 \mathrm{~mm}$; $\mathrm{NaHCO}_{3}, 3 \mathrm{~mm}$, HEPES (pH 7.4), $5 \mathrm{~mm}$; penicillin $\mathrm{G}, 5 \mathrm{U} / \mathrm{ml}$; streptomycin, $5 \mu \mathrm{g} / \mathrm{ml}$; and $8 \%$ heat-inactivated fetal calf serum (Flow Laboratories). The cells were kept for 3 weeks with 2 changes of culture medium each week. After 3 weeks, the cultures consisted of astrocyte monolayers, as confirmed by immunocytochemistry using anti-glial fibrillary acidic protein and anti-neurofilament antisera (Denis-Donini et al., 1984).

Freshly dissociated mesencephalic and striatal cells from 15- and 16d-old rat embryos, respectively (except as noted) were seeded on the astrocytic monolayers at densities varying from $2.5 \times 10^{3}$ to $10^{4}$ cells/ $\mathrm{cm}^{2}$ and further cultured for 2 or $5 \mathrm{~d}$ in a synthetic medium supplemented with a mixture of proteins, hormones, and salts (Di Porzio et al., 1980; Prochiantz et al., 1982).

In some cases mesencephalic cells were directly seeded on polyornithine-coated coverslips and cultured in the chemically defined medium.

Preparation of MAP2 antiserum. MAPs were separated from purified rat brain tubulin by thermodenaturation in high salt (Fellous et al., 1977). After electrophoresis of MAPs on a $4-15 \%$ linear gradient of polyacrylamide in the presence of SDS, the bands corresponding to MAP2 were excised and used for the production of MAP2 antiserum by albino rabbits. The specificity of the antibody was checked by solidphase radioimmunoassay, immunoblotting of whole brain extracts, and immunostaining of cerebellar sections.

Immunocytochemistry. The coverslips were immersed for $1 \mathrm{hr}$ at $4^{\circ} \mathrm{C}$ in freshly prepared $4 \%$ paraformaldehyde in PBS. After 3 washes with PBS containing $10 \%$ fetal calf serum and $0.1 \%$ Triton X-100 (buffer A), the cells were incubated $1 \mathrm{hr}$ at $37^{\circ} \mathrm{C}$ with MAP2 antiserum diluted 1:400 in buffer $A$. The coverslips were washed 3 times with buffer $A$ and further incubated for $1 \mathrm{hr}$ at $37^{\circ} \mathrm{C}$ with peroxidase-conjugated goat anti-rabbit IgG (1:100, Amersham). The presence of the immunocomplex was revealed after 3 more washes in buffer $A$ by a short incubation at room temperature with $0.003 \% \mathrm{H}_{2} \mathrm{O}_{2}$ and diaminoben zidine, $0.5 \mathrm{mg}$ $\mathrm{ml}$ (Sigma), in Tris $\mathrm{HCl}, 50 \mathrm{~mm}, \mathrm{pH} 7.4$.

Double-staining experiments with anti-MAP2 antiserum and a mouse monoclonal anti-MAPl antibody (kindly provided by Dr. David Asai) was done as above, except that the complexes were visualized with rhodamine-conjugated goat anti-rabbit and fluorescein-conjugated goat anti-mouse IgG (Amersham), respectively.

\section{Results}

\section{Quantitative analysis of MAP2-positive neurons}

Neurons were considered to be MAP2 positive if any segment in the cell body, neurite, or arborization was strongly stained. However, staining was rarely present uniformly on the cell. Figure I shows some examples of stained and unstained neurons. The percentages of MAP2-positive and MAP2-negative mesencephalic (mes) and striatal (str) neurons on mesencephalic
Table 1. Percentage of MAP2-positive neurons in different neuroastroglial coculture conditions

Percentage MAP2-positive neurons mes/Gmes mes/Gstr str/Gmes str/Gstr

\begin{tabular}{rllll}
\hline $\begin{array}{r}\text { Embryonic glia } \\
\text { Experiment 1 }\end{array}$ & & & & \\
$\mathrm{E}^{15}+2$ div & $80 \pm 7$ & $54 \pm 7$ & $28 \pm 9$ & $71 \pm 10$ \\
Experiment 2 & & & & \\
$\mathrm{E}^{15}+2$ div & 74 & 38 & 13 & 58 \\
$\mathrm{E}^{15}+5 \mathrm{div}$ & 91 & 55 & 24 & 79 \\
$\mathrm{~F}^{18}+2$ div & 75 & 54 & 39 & 58 \\
Postnatal glia & & & & \\
Experiment 3 & & & & \\
E $^{15}+2$ div & 71 & 29 & 24 & 85
\end{tabular}

Embryonic and postnatal astrocytes were prepared from 15-d-old embryos and postnatal day 4 animals, respectively, and grown for 3 weeks. Neuronal age can be determined by adding the age of the embryos from which the cells were prepared and the number of days in vitro (div).

(Gmes) and striatal (Gstr) astrocytes were then determined for each condition. Results shown in Table 1 for experiment 1 were obtained at $2 \mathrm{~d}$ of coculture in serum-free medium with 14-dold (mes) or 15-d-old (str) embryonic neurons plated at low density $\left(5 \times 10^{3}\right.$ cells $\left./ \mathrm{cm}^{2}\right)$ on the astroglial monolayers. Under these conditions, neurons were sufficiently isolated to be examined scparately and could therefore be fully characterized morphologically. The data from Table 1 (experiment 1) were collected from 4 independent experiments in which 100 randomly selected neurons were examined. The percentage of MAP2-positive cells was always higher in homotopic (mes/Gmes, $\mathrm{str} / \mathrm{Gstr}$ ) than in heterotopic condition (mes/Gstr, str/Gmes); these differences were statistically significant (Student's $t$ test, $p \leq 0.01$ ). The differences between homotopic and heterotopic conditions were more pronounced with the striatal than with the mesencephalic neurons because of the small number of positive striatal neurons in heterotopic neuro-astroglial cocultures.

Several conditions that could modulate the expression of MAP2 have been assessed: (1) the age of the neurons, (2) the time in culture, (3) the age of the animals from which glial monolayers were prepared, and (4) the density of neuronal plating. Typical results are presented in Table 1 (experiments 2 and 3). Astroglial cells prepared from postnatal day 4 (P4) gave the same results as those prepared from 15-d-old embryos, indicating that this was not an important factor. Time in culture had a marginal influence by increasing the number of positive cells in heterotopic cocultures. The same kind of increase was observed when neurons were prepared from 18-d-old instead of 15-d-old embryos. The most important factor was the initial neuronal plating density. It was found that the cells started to express MAP2 independently of the homotopic or heterotopic nature of the substratum, and even on a polyornithine synthetic substratum at plating densities above $10^{4} \mathrm{cells} / \mathrm{cm}^{2}$. This might be due to autoconditioning of the medium by the neurons and to the establishment of neuroneuronal contacts. In synthetic medium, however, while the percentage of non-neuronal cells was less than $5 \%$, at high plating densities their absolute number could be sufficient to promote MAP2 expression. To avoid this complication and also to provide conditions for best analysis of the neurons, all experiments were done at low density (2.5$5 \times 10^{3}$ cells $/ \mathrm{cm}^{2}$ ). 

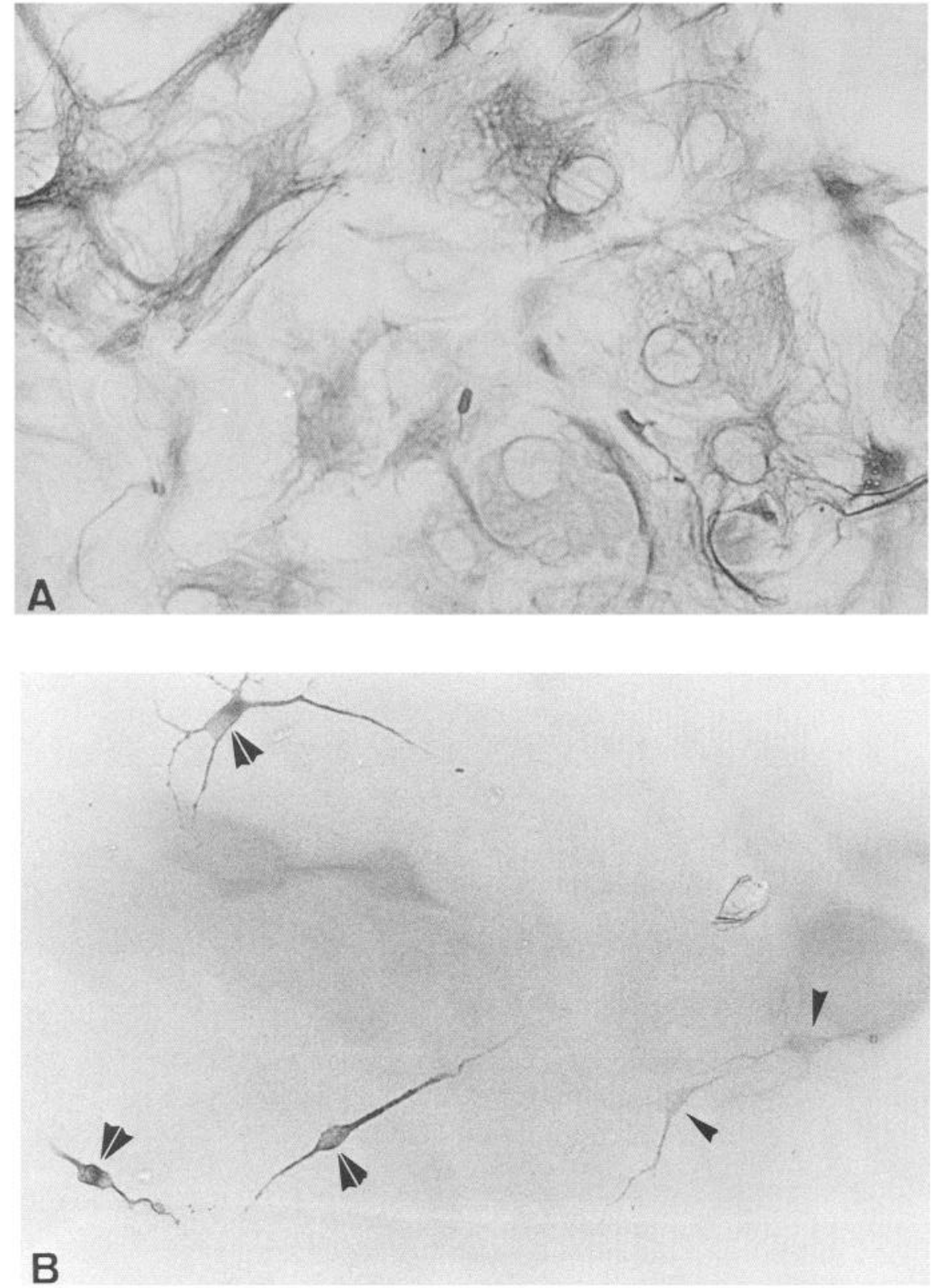

Figure 1. Immunostaining of astrocytes and neurons with anti-GFAP and anti-MAP2 antibodies. $A$, Aspect of an astrocytic monolayer stained with an antibody directed against the glial fibrillary acidic protein. $\times 1300$. $B$, Example of MAP2-positive ( $\mathrm{dou}$ ble arrows) and MAP2-negative neurons (single arrows). $\times 1300$.

\section{Morphological analysis of MAP2-negative and MAP2-positive neurons}

In order to assess whether MAP2 expression was associated with specific morphological traits, the morphologies of MAP2negative and MAP2-positive neurons were compared. In these experiments, 100 positive and negative cells were randomly selected, drawn, and classified according to the number of neurites originating from the cell body (primary neurites). From these drawings histograms were constructed comparing (1) the number of primary neurites and (2) the number of branching points per neuron per class $(0,1,2,3,4$, or more primary neurites).

The results obtained in one of these experiments are shown in Figure 2 (number of primary neurites) and Figure 3 (number of branching points per neuron per neuronal class). In homotopic and heterotopic coculture conditions, MAP2-negative cells had few primary neurites (0-2) and no branching points. Neurons stained with MAP2 had more primary neurites, which often produced secondary and tertiary branches.

\section{Comparison between MAP2-positive cells in homotopic and heterotopic neuro-astroglial cocultures}

The drawings of the MAP2-positive neurons also allowed us to compare the morphologies of MAP2-positive neuronal populations in both homotopic and heterotopic cocultures. Figure 4 illustrates one experiment in which 100 randomly selected MAP2-positive neurons were drawn after $2 \mathrm{~d}$ in culture. With regard to either mesencephalic or striatal neurons, it is clear that neurons having 3,4 , or more neurites are more numerous on homotopic (Fig. 4, $A, C$ ) than on heterotopic astrocytes (Fig. $4, B, D)$. This finding has been consistently observed in several independent experiments and a quantification for one of these experiments is presented in Figure 2 (hatched bars). The number 

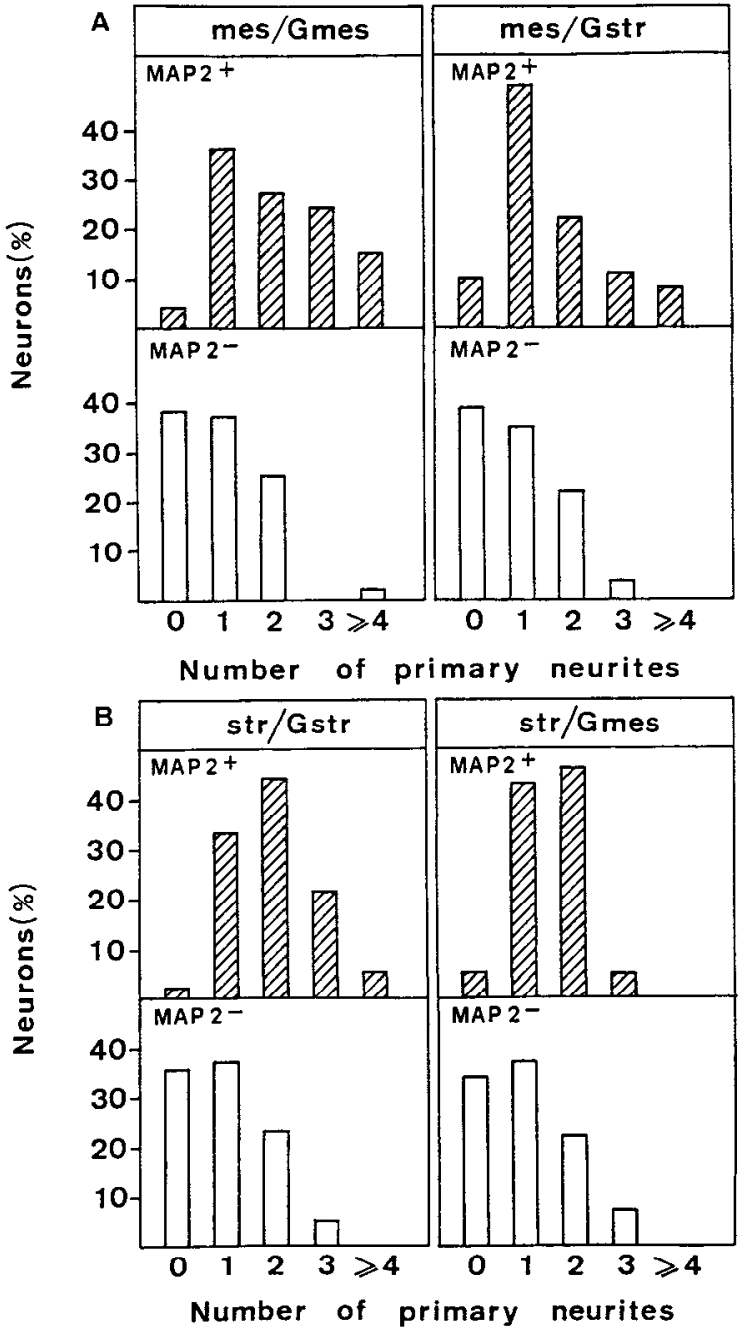

Figure 2. Distribution according to the number of primary neurites of MAP2-positive and MAP2-negative mesencephalic and striatal neurons in homotopic and heterotopic neuro-astroglial cocultures. For each panel 100 MAP2-positive and 100 MAP2-negative neurons were randomly selected and drawn as in Figure 5. Hatched bars, MAP2-positive neurons; empty bars, MAP2-negative neurons.

of branching points was also increased in homotopic versus heterotopic cocultures. This latter point was clearer with mesencephalic than with striatal neurons (Fig. 3, hatched bars). Therefore, we conclude that MAP2-positive neurons have a higher number of primary neurites in homotopic than in heterotopic neuro-astroglial cocultures. In addition, a higher number of branching points is observed in MAP2-positive mesencephalic neurons grown in homotopic conditions.

\section{Distribution of MAP2 staining}

As already stated, any neuron that showed some staining, either at the level of its cell body or in some part of its arborization, was counted positive, and cells that were completely stained, or even uniformly labeled, were rare. This is demonstrated by comparing immunostaining and phase contrast (Fig. 5, $A, B$ ) and even more clearly by double-labeling experiments with antibodies directed against MAP2 and MAP1, another microtubule-associated protein that is not enriched in dendrites (Bloom et al., 1984; Huber and Matus, 1984). Indeed, not all areas

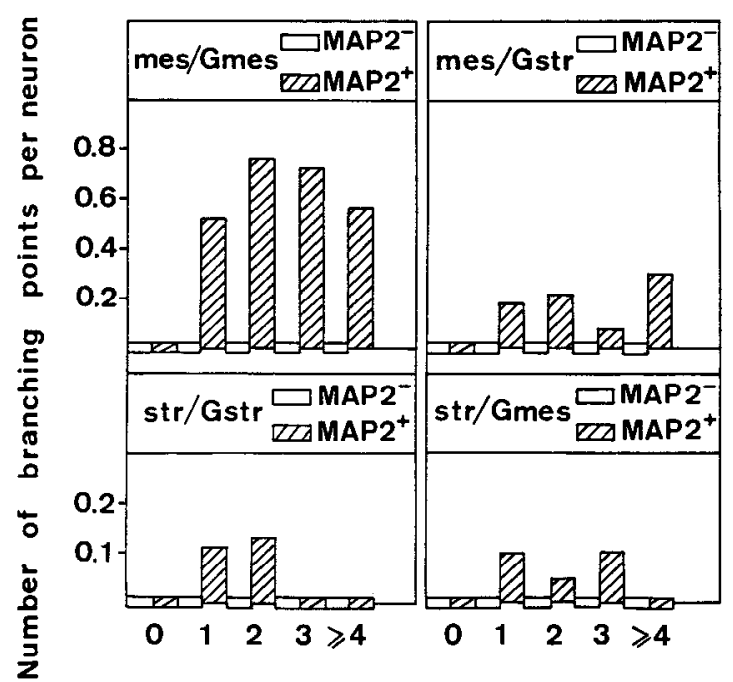

Number of primary neurites

Figure 3. Number of branching points per neuron compared for MAP2positive and MAP2-negative mesencephalic and striatal neurons in homotopic and heterotopic neuro-astroglial coculture. This number was calculated as a function of the number of primary neurites. For each panel, 100 MAP2-positive and 100 MAP2-negative neurons were randomly selected and drawn as in Figure 5.

labeled with anti-MAP1 also stain with anti-MAP2 (Fig. 5, $C$, D).

Since MAP2 has been reported to be associated mainly with the dendritic compartment, we have studied the cellular distribution of the antigen and analyzed the branching of MAP2positive versus MAP2-negative neurites. This was done for the mesencephalic and striatal neurons in homotopic and heterotopic cocultures. Figure 5 illustrates an example of a neuron having MAP2-positive and MAP2-negative neurites. Some neurites that are largely MAP2 negative (and therefore considered as such) nevertheless may have some faintly labeled varicosities disposed more or less regularly along the neurite (Fig. $5 \mathrm{~A}$ ). Negative neurites can grow directly from the cell body, be in continuity with a heavily stained segment, or depart from one strongly labeled neurite. Most of them are long, thin, and straight, with few branching points, and represent the major neuritic population under heterotopic neuro-astroglial coculture conditions. MAP2-positive neurites are usually shorter and thicker. The staining is generally more intense in the vicinity of the cell body and appears to fade as the neurite tapers; these neurites seem to present more branching points than MAP2-negative neurites and to be more numerous in homotopic neuro-astroglial cocultures.

We have quantified the number of branching points in the heavily and lightly stained neurites. The branching points were classified into 3 categories, as schematized in Figure 6. Either the branches (mother and daughter branches) had the same staining intensity or one or more of these branches was less intensely stained. The differences between the total number of branching points in homotopic and heterotopic cocultures can be attributed to the high branching ability of the heavily stained neurites.

\section{Discussion}

In this paper we report that both the expression of cytoskeletal protein MAP2 and the shape of brain neurons can be regulated 


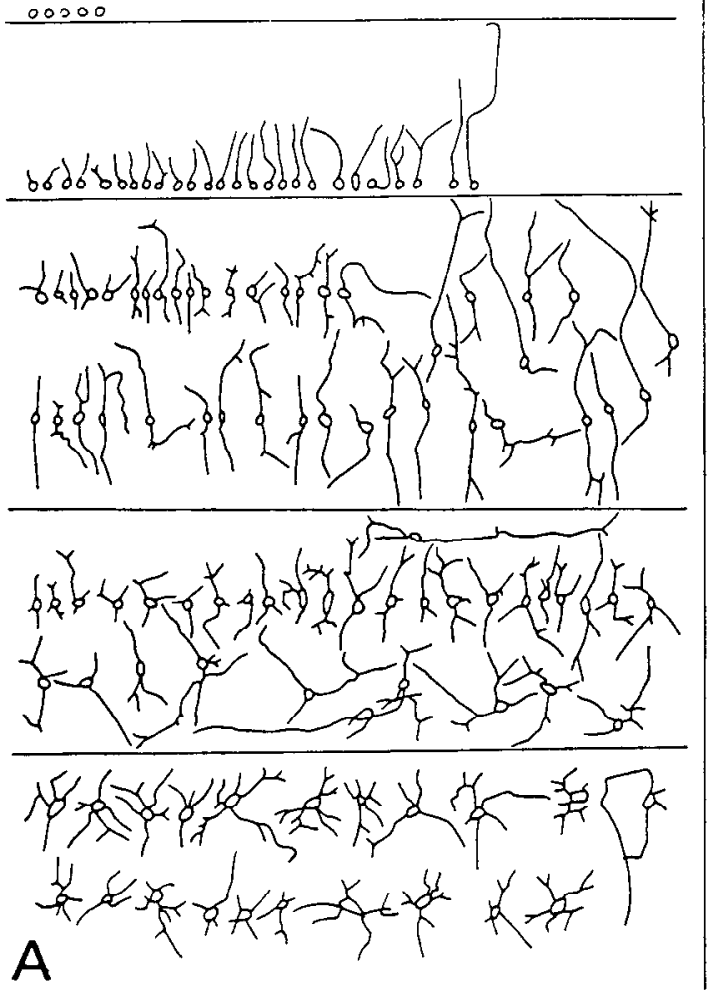

00000000000000000
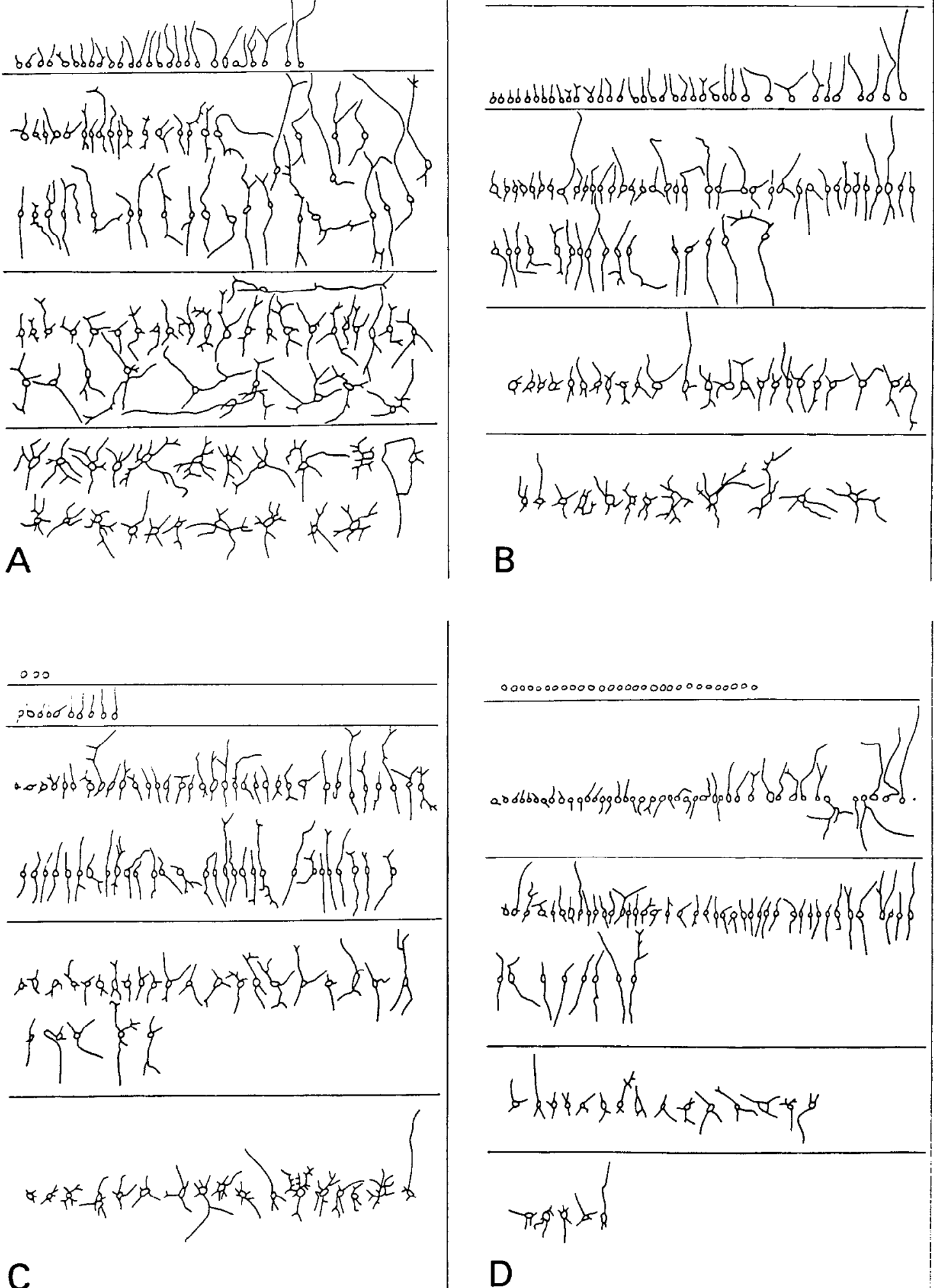

Figure 4. Drawings of MAP2-positive mesencephalic and striatal neurons in homotopic and heterotopic neuro-astroglial cocultures. Stained neurons were taken at random and drawn with the help of a light chamber and classified according to the number of primary neurites $(0,1,2,3$, 24). $A$, mes/Gmes; $B$, mes/Gstr; $C$, str/Gstr; $D$, str/Gmes. $\times 75$. 

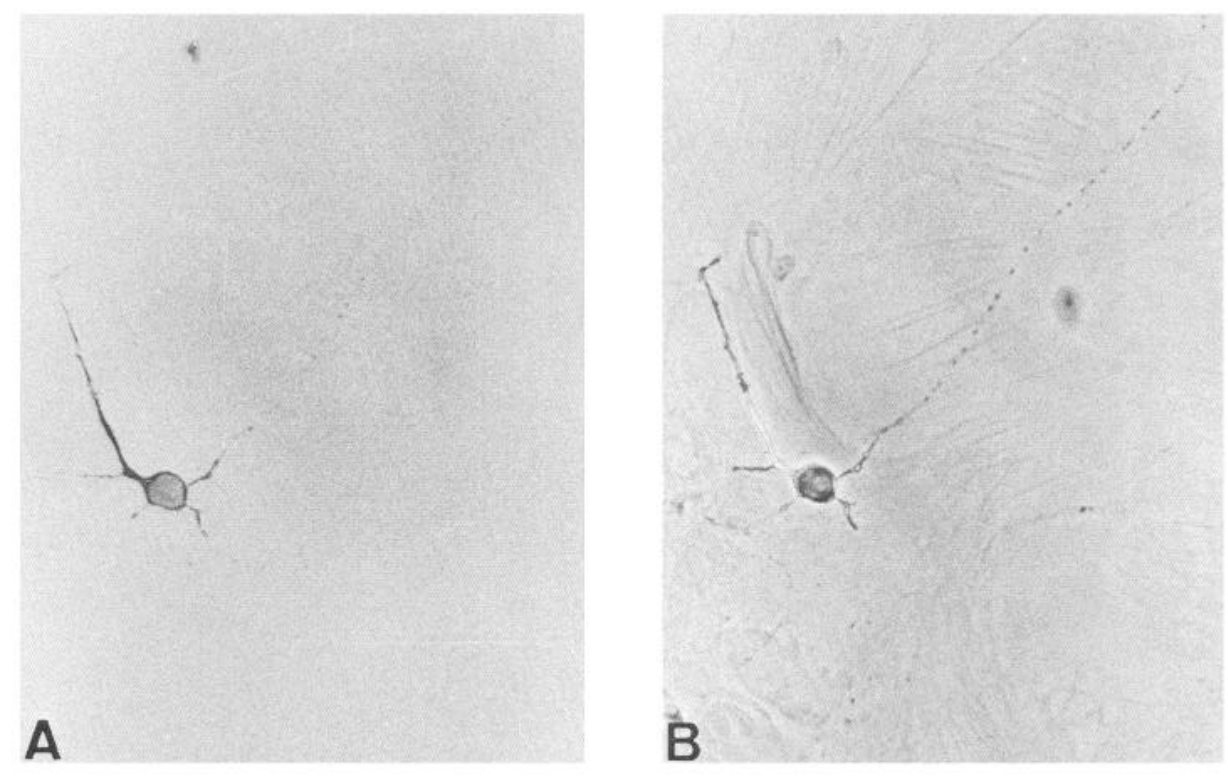

Figure 5. Immunostaining demonstration of heterogenous distribution of MAP2. A, Striatal neuron on striatal astrocytes labeled with anti-MAP2 antibodies. $B$, Same field as in $A$. Phase contrast. $\times 1500$. $C$ and $D$, Mesencephalic neurons on mesencephalic astrocytes double-stained with anti-MAP2 $(C)$ and anti-MAP1 $(D)$ antibodies. $\times 1300$.
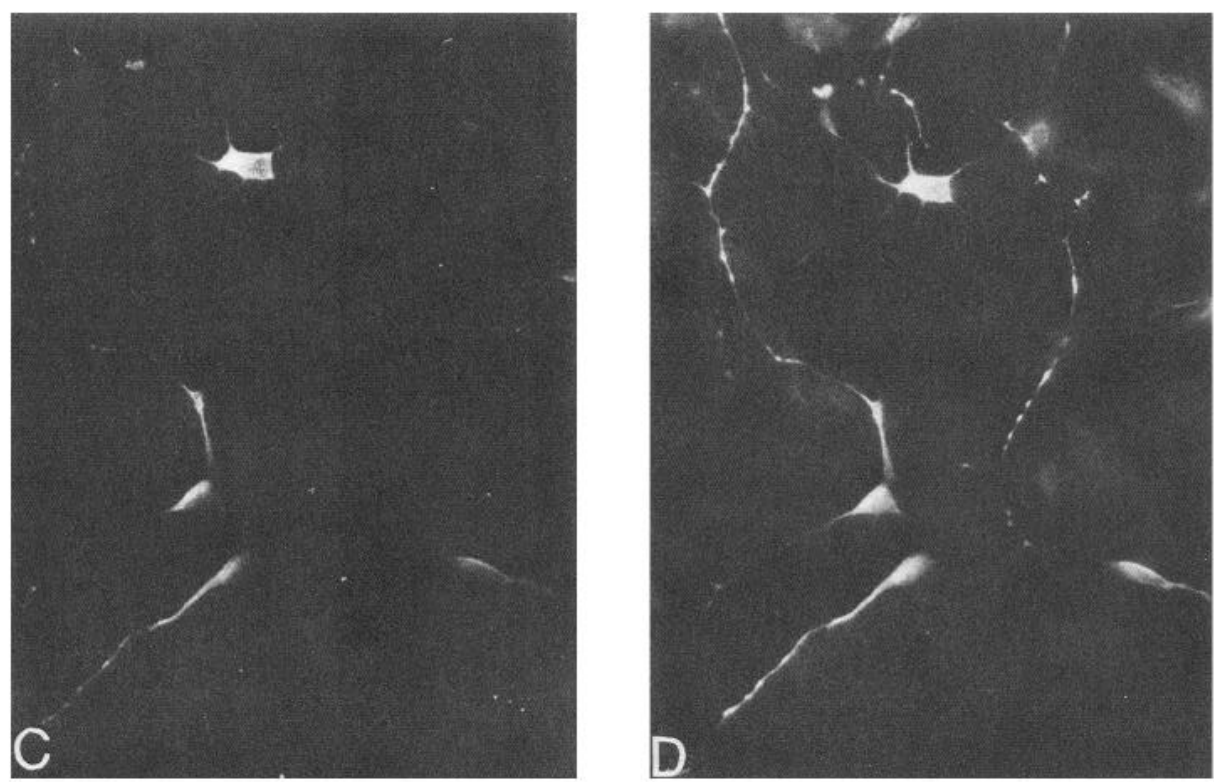

in culture by the glial environment. Specifically, neurons from 2 different brain regions (the mesencephalon and the striatum) exhibit enhanced MAP2 staining and produce more branching primary neurites when plated on homotopic than on heterotopic astrocytes. In addition, neurites that are strongly positive for MAP2 exhibit enhanced branching ability.

The results confirm our previous observations on mesencephalic dopaminergic neurons and extend them to other nondopaminergic and striatal neuronal populations (Denis-Donini et al., 1984). Although such a regulatory effect of in vitro environmental conditions on neuritic morphology and MAP2 expression has already been reported (Allen and Higgins, 1986; Bruckenstein and Higgins, 1986; Fischer et al., 1986), the importance of region-specific interactions has seldom been examined.

The specificity of the morphogenetic influence of non-neuronal cells on neuronal development was suggested by Mudge (1984), however, who showed that the typical T-shape of the sensory neuron arborization is obtained in culture only with the addition of Schwann cells and not with fibroblasts, astrocytes, or various other cell lines. As reported here, it appears that, even within a single class of non-neuronal cell, regional variations exist that are important to the specificity of the interaction.

This specific effect of the homotopic conditions is demonstrated by the fact that the overall complexity of all striatal and mesencephalic neurons is higher in homotopic than in heterotopic neuro-astroglial cocultures. Among these neurons the MAP2-positive neurons are more numerous in homotopic cocultures and have more primary neurites and branching points than the MAP2-negative cells. Finally, the MAP2-positive neurons present in homotopic cocultures are morphologically more complex than those present in heterotopic cultures.

The expression of MAP2 normally increases with neuronal maturity (Crandall et al., 1986). Indeed, even in heterotopic conditions the number of MAP2-positive cells increases with time in vitro or with the age of the embryos from which the 


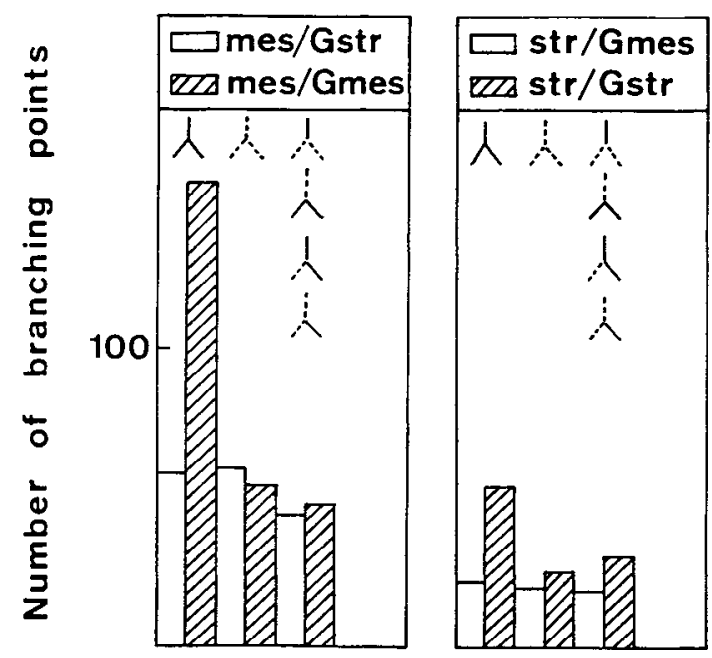

Figure 6. Number of branching points for MAP2-positive and/or MAP2-negative neurites compared in homo- and heterotopic neuroastroglial cocultures. Each panel is the result of the examination of 100 neurons taken at random and drawn with the help of a light chamber. For each neuron, heavily stained $(-)$ and lightly stained $(--)$ neurites were distinguished. This allowed us to classify the branching points into the 3 categories shown.

neurons are prepared. Therefore, the increased percentage of MAP2-positive neurons in homotopic versus heterotopic neuroastroglial cocultures very likely reflects a region-specific maturational effect of the astrocytes. Measurement of absolute quantities of neuronal MAP2 would seem a more direct test, but astroglial cells, while never stained with MAP2 antibody under our conditions (Figs. 1, 5), nevertheless synthesized this antigen, as demonstrated by immunoprecipitation (not shown) and as reported by Bloom et al. (1984) and Couchie et al. (1985). Therefore, variations in coculture could not have been attributed with certainty to the neuronal compartment.

The shape of the arborization and the distribution of MAP2 suggest that homotopic coculture conditions might favor the development of a dendrite-like compartment. Since it has been consistently reported that MAP2 is a rather specific dendritic marker (Matus et al., 1981; Caceres et al., 1984; De Camilli et al., 1984), the increase in MAP2 staining could therefore reflect an increase in the growth of the dendrites. One must remain cautious in making this interpretation, however, since Caceres et al. (1986) have shown that MAP2 is present in immature axons and is later redistributed into the dendritic compartment. Nonetheless several lines of evidence indicate that, in our culture conditions, MAP2 accumulates in a dendrite-like compartment that develops faster in homotopic than in heterotopic neuroastroglial cocultures. First, MAP2 is unevenly distributed within the cell, as shown by the double labeling with anti-MAPl and -MAP2 antibodies and by comparing phase-contrast pictures to immunostaining with MAP2. Second, cells that do not present any MAP2 immunoreactivity have no branching points. Third, the additional branching points that appear in homotopic cocultures are specifically associated to neurites most heavily stained by MAP2 antibodies. Despite these data, the definitive demonstration that homotopic neuro-astroglial coculture conditions favor the outgrowth of MAP2-positive dendrites will require direct observation of the MAP2-containing neurites at the ultrastructural level. These experiments are presently in progress.
The molecular mechanisms leading to enhanced MAP2 expression and outgrowth of a dendrite-like compartment are unknown. Among several hypotheses, one may postulate the involvement of region-specific neuronotrophic factors. Such a possibility is supported by the fact that NGF promotes the in vitro maturation of central cholinergic neurons and presents a specific distribution in the brain (Honneger and Lenoir, 1982; Hefti et al., 1985; Korshing et al., 1985; Martinez et al., 1985; Levi-Montalcini and Aloe, 1986; Witthmore et al., 1986).

The possible involvement of local neuronotrophic factors does not preclude other mechanisms. Among these, one can postulate the existence of local isoforms of cell and substratum adhesion molecules generated by alternative splicing or posttranslational modification. In some cases, the regulation of surface molecule synthesis or modification could even be under the control of a soluble factor, as suggested by the recent discovery that NILE (NGF-inducible large external protein), neuron-glia adhesion molecule ( $\mathrm{NgCAM}$ ), and surface glycoprotein $\mathrm{L} 1$ are probably identical (Bock et al., 1985; Friedlander et al., 1986; Sajovic et al., 1986).

\section{References}

Allen, M., and D. M. Higgins (1986) Ganglionic non-neural cells induce dendritic growth in cultured sympathetic neurons. Soc. Neurosci. Abstr. 12: 149.

Bloom, G. S., T. A. Schoenfeld, and R. B. Vallee (1984) Widespread distribution of the major polypeptide component of MAP 1 (microtubule associated protein 1) in the nervous system. J. Cell Biol. 98. 320-330.

Bock, E., C. Richter-Landsberg, A. Faissner, and M. Schachner (1985) Demonstration of immunological identity between the nerve growth factor-inducible large external (NILE) glycoprotein and the cell adhesion molecule L1. EMBO J. 4: 2765-2768.

Bruckenstein, D., and D. Higgins (1986) Environmental determinants of dendritic growth in cultured sympathetic neurons. Trans. Am. Soc. Neurochem. Abstr. 17: 197 (abstr.).

Caceres, A., G. Banker, O. Steward, I. Binder, and M. Payne (1984) MAP-2 is localised to the dendrites of hippocampal neurons which develop in culture. Dev. Brain Res. 13: 314-318.

Caceres, A., G. A. Banker, and L. Binder (1986) Immunocytochemical localization of tubulin and microtubule-associated protein 2 during the development of hippocampal neurons in culture. J. Neurosci. 6 : $714-722$.

Chamak, B., A. Fellous, A. Autillo-Touati, G. Barbin, and A. Prochiantz (1987) Are neuro-astroglial neuronotrophic interactions regionally specified? Ann. NY Acad. Sci. (in press).

Chiquet, M., and S. E. Acklin (1986) Attachment to ConA or extracellular matrix initiates rapid sprouting by cultured leech neurons. Proc. Natl. Acad. Sci. USA 83: 6188-6192.

Couchie, D., C. Fages, A. M. Bridoux, B. Rolland, M. Tardy, and J. Nunez (1985) Microtubule-associated proteins (MAPs) and in vitro astrocyte differentiation. J. Cell Biol. 101: 2095-2103.

Crandall, J. E., M. Jacobson, and K. S. Kosik (1986) Ontogenesis of microtubule-associated protein 2 (MAP2) in embryonic mouse cortex. Dev. Brain Res. 28: 127-133.

De Camilli, P., P. E. Miller, F. Navone, W. E. Theurkauf, and R. B. Vallee (1984) Distribution of microtubule-associated protein 2 in the nervous system of the rat studied by immunofluorescence. Neuroscience 11: 819-846.

De George, J. J., and S. Carbonetto (1986) Wheat germ agglutinin inhibits nerve fiber growth and concanavalin A stimulates nerve fiber initiation in culture of dorsal root ganglia neurons. Dev. Brain Res. 28: $169-175$.

Denis-Donini, S., J. Glowinski, and A. Prochiantz (1984) Glial heterogeneity may define the three-dimensional shape of mouse mesencephalic dopaminergic neurones. Nature 307: 641-643.

Di Porzio, U., M.-C. Daguet, J. Glowinski, and A. Prochiantz (1980) Effect of striatal cells on in vitro maturation of mesencephalic dopaminergic neurones grown in serum-free conditions. Nature 288: $370-373$. 
Edgar, D., R. Timpl, and II. Thoenen (1984) The heparin-binding domain of laminin is responsible for its effects on neurite outgrowth and neuronal survival. EMBO J. 3: 1463-1468.

Fellous, A., J. Francon, A. M. Lennon, and J. Nunez (1977) Microtubule assembly in vitro. Purification of assembly-promoting factors. Eur. J. Biochem. 78: 167-174.

Fischer, I., J. B. Shea, V. S. Sapirstein, and K. S. Kosik (1986) Expression and distribution and microtubule-associated protein 2 (MAP2) in neuroblastoma and primary neuronal cells. Dev. Brain Res. 25: 99-109.

Friedlander, D. R., M. Grumet, and G. M. Edelman (1986) Nerve growth factor enhances expression of neuron-glia cell adhesion molecule in PC12 cells. J. Cell Biol. 102: 413-419.

Grumet, M., U. Rutishauser, and G. M. Edelman (1983) Neuron-glia adhesion is inhibited by antibodies to neural determinants. Science 222: $60-72$.

Grumet, M., S. Hoffman, K. L. Crossin, and G. E. Edelman (1985) Cytotactin, an extracellular matrix protein of neural and non-neural tissues that mediates glia-neuron interaction. Proc. Natl. Acad. Sci. USA 82: 8075-8079.

Guenter, J., N. Hanspeter, and D. Monard (1985) A glia-derived neurite-promoting factor with protease inhibitory activity. EMBO J. 4: 1963-1966.

Hefti, F., J. Hartikka, F. Eckenstein, H. Gnahn, R. Heumann, and M. Schwab (1985) Nerve growth factor increases choline acetyltransferase but not survival or fiber outgrowth of cultured fetal septal cholinergic neurons. Neuroscience 14: 55-68.

Honneger, P., and D. Lenoir (1982) Nerve growth factor (NGF) stimulation of cholinergic telencephalic neurons in aggregating cell cultures. Dev. Brain Res. 3: 229-238.

Huber, G., and A. Matus (1984) Differences in the cellular distribution of two microtubule-associated proteins, MAP1 and MAP2 in rat brain. J. Neurosci. 4: 151-160.

Kligman, D., and D. R. Marshak (1985) Purification and characterization of a neurite extension factor from bovine brain. Proc. Natl. Acad. Sci. USA 82: 7136-7139.

Korshing, S., G. Auburger, R. Heumann, J. Scott, and H. Thoenen (1985) Levels of nerve growth factor and its mRNA in the central nervous system of the rat correlate with cholinergic innervation. EMBO J. 4: 1389-1393.

Kruse, J., G. Keilhauer, A. Faissner, R. Timpl, and M. Schachner (1985) The J1 glycoprotein, a novel nervous system cell adhesion molecule of the L2/HNK-1 family. Naturc 316: 146-148.

Lander, A. D., D. K. Fujii, and L. F. Reichardt (1985) Laminin is associated with the "ncuritic outgrowth-promoting factors" found in conditioned media. Proc. Natl. Acad. Sci. USA 82: 2183-2188.

Levi-Montalcini, R. (1982) Developmental neurobiology and the natural history of nerve growth factor. Annu. Rev. Neurosci. 5: 341362.

Levi-Montalcini, R., and L. Aloe (1985) Differentiating effects of murine nerve growth factor in the peripheral and central nervous system of Xenopus laevis tadpoles. Proc. Natl. Acad. Sci. USA 82: 71117115 .

Martinez, H. J., C. F. Dreyfus, G. M. Jonakait, and I. B. Black (1985) Nerve growth factor promotes cholinergic development in brain striatal cultures. Proc. Natl. Acad. Sci. USA 82: 7777-7781.

Matus, A., R. Bernhardt, and T. Hugh-Jones (1981) High molecular weight microtubule-associated proteins are preferentially associated with dendritic microtubules in brain. Proc. Natl. Acad. Sci. US $\Lambda 78$ 3010-3014.

Mudge, A. W. (1984) Schwann cells induce morphological transformation of sensory neurons in vitro. Nature 309: 367-369.

Peng, I., L. I. Binder, and M. M. Black (1986) Biochemical and immunological analyses of cytoskeletal domains of neurons. J. Cell Biol. 102: 252-262.

Prochiantz, A., A. Delacourte, M.-C. Daguet, and D. Paulin (1982) Intermediate filament proteins in mouse brain cells cultured in the presence or absence of fetal calf serum. Exp. Cell Res. 139: 404-410.

Rutishauser, U. (1983) Molecular biological properties of a neural cell adhesion molecule. Cold Spring Harbor Symp. Quant. Biol. 48: 501514.

Sajovic, P., E. Kouvelas, and E. Trenkner (1986) Probable identity of NILE glycoprotein and the high-molecular-weight component of L1 antigen. J. Neurochem. 47: 541-546.

Smalheiser, N. R., S. M. Crain, and L. M. Reid (1984) Laminin as a substrate for retinal axons in vitro. Dev. Brain Res. 12: 136-140.

Stallcup, W. B., and L. Beasley (1985) Involvement of the NGF inducible large external glycoprotein (NILE) in neurite fasciculation in primary cultures of rat brain. Proc. Natl. Acad. Sci. USA 82: 12761280.

Walicke, P., W. M. Cowan, N. Veno, A. Baird, and R. Guillemin (1986) Fibroblast growth factor promotes survival of dissociated hippocampal neurons and enhances neurite extension. Proc. Natl. Acad. Sci. USA 83: 3012-3016.

Witthmore, S. R., T. Ebendal, L. Lärkfors, L. Olson, A. Seiger, I. Strömberg, and H. Persson (1986) Developmental and regional expression of $\beta$-ncrve growth factor messenger RNA and protein in the rat central nervous system. Proc. Natl. Acad. Sci. USA 83: 817-821. 